\title{
The Fabrication Method of Electrical Thermal Mask with High Performance Filtration and Functions
}

\author{
Kou-Bin Cheng* \\ ${ }^{1}$ Department of Fiber and Composite Materials, Feng Chia University, Taiwan \\ ${ }^{2}$ Textile and Material Industrial Research Center, Feng Chia University, Taiwan
}

Submission: January 01, 2018; Published: January 23, 2018

*Corresponding author: Kou-Bin Cheng, Department of Fiber and Composite Materials, Textile and Material Industry Research Center, Feng Chia University, 100, Wen Hua Rd, Sea Tween District, Taichung 407, Taiwan; Email: kbcheng@fcuoa.fcu.edu.tw

\begin{abstract}
In order to overcome the serious allergens, dust mite and epidemics healthy problems, the electrical thermal mask with high performance filtration and functions has been proposed in this paper. The electrical thermal mask belongs to one of the smart textile applications which is laminated and sewed together from a multi-layers integrated structure and thermal generated part to achieve the bacteria, powders, viral filtration efficiency, and due to thermal electrical effects, which produce the warm retention functions and overcome the nose allergens. The functions of mask also have air permeability, comfort, light and soft, fare infrared radiation, ultra-performance filtration, and moisturizing etc. Furthermore, it can re use, easy storage, durability, health care, washable, safety, and electrical warning advantages.
\end{abstract}

Keywords: Electrical thermal mask; Bacteria and viral filtration efficiency; PTFE nano Fiber Membrane; Thermal conductive film

\section{Introduction}

Nowadays, people's living is seriously influenced by allergens, dust mite and epidemics caused by thermal power generation, fuel emissions and other reasons. Medical research has shown that particles size less than $2.5 \mu \mathrm{m}$ will penetrate deeply into the pulmonary alveoli of the lung through the mouth and nose, and further diffuse into the organs of our body through the blood vessels, resulting in a risk of lung cancer, dementia, hypertension, stroke, heart disease, asthma, even obesity. The purpose of this paper is to propose an improvement in people's breathing quality to maintain the respiratory system in a healthy state, by developing a device which could be used to protect and take good care of the respiratory system positively. A solution is proposed with the joint cooperation of industry and academy by a combination of the high efficiency air filtration, electrical thermal heating and LED lighting element and functions. This is achieved by using a 3D mask with high filtration efficiency embedded with an effective electrical thermal heating pad using a graphene thin film with super heat transfer characteristics attached on the bridge of nose position. With its efficient filtration, clean air is inhaled and then warmed up by the heating pad, thus comfortable warm air is produced for the nose, mouth and respiratory system. The LED lighting element is equipped onto bridge of nose position outside which blinking display to show the exact position for safety. The products are belonging to the one of intelligent textile applications which 3D structure have air permeability comfort, light and soft, fare infrared radiation, ultra-performance filtration, moisturizing, safety, and other functions. It has reuse, easy storage, durability, health care, washable, and safety electrical warning advantages [1-5].

\section{Experiment}

Figure 1 shows a schematic diagram of a 3 dimensional electrical thermal mask, the mask structure has included 1: Mask body, 2: Inner Fabric, 3: Outer Fabric, 4: Ear Hangers, 5: Connected wire, 6. Filtrate Nanofiber Membrane, 7: Air permeability fabric, 8: First layer thread, 9. Second layer thread, 10: Interlayer, 11: Thermal conductive film, 12: Mini USB, 13: Power source, and 14: Connected wire. Which are laminated and sewed together to provide a multi- layers integrated structure to achieve the bacteria, powders, viral filtration efficiency, and due to thermal electrical effect which produce the warm retention functions. The inner fabric of mask is made from polyester circular knitted fabrics made with moisture transfer function, absorb saliva and perspiration. The outer fabric is made from polyester circular knitted fabric with far infrared functions and can be printed in various fashion patterns. The filtration nonwoven is fabricated from Polytetra Fluoro Ethylene (PTFE) nano fiber membrane with $250-420 \mathrm{~nm}$ fiber fineness by using an electrostatic sinning method. Electro spinning is a unique technique able to produce nanofibers and continuous filaments with nanometer size diameter, which are applicable in various fields such as biomedical, 
filtration, healthcare, biotechnology, defense and security, environmental engineering and energy generation etc. Among the various electro spun polymer systems, Poly Tetra Fluoro Ethylene (PTFE) has been widely investigated due to its unique chemical resistance properties, namely corrosion resistance, high and low temperature resistance, electrical insulation, selflubricating, surface non-sticky, resistance to atmospheric aging, non-flammable (Limiting oxygen index (LOI)<90), and excellent mechanical properties, good thermal stability and processability etc. The PTFE nanofiber membrane is most important in this paper in improving the bacteria, powders less than $2.5 \mu \mathrm{m}$ and viral filtration efficiency. The air permeability, comfort, pressure difference, and other performance of 3D electrical thermal mask is better than N95 and N99 mask. Ear hangers are produced from the elastic band for narrow width wearing technology easily. The side view of the mask body which included first layer thread, second layer thread, interlayer, and thermal conductivity film to achieve better thermal conductive and diffusivity performance as well as the uniform thermal distributions [1-3].

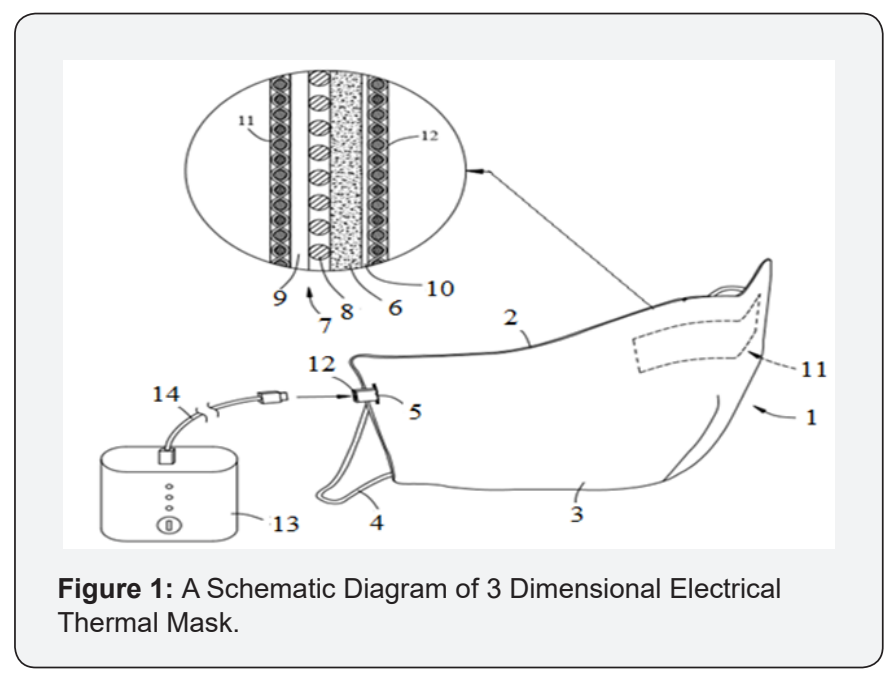

\section{Results and Discussion}

The filter media of this novel mask has been certificated by Nelson Lab. of the USA, both of the bacteria filtration efficiency and viral filtration efficiency are $99.9 \%$ and also tested with different particle size of between 7.0-0.4 micron by TTRI in Taiwan, the filtration efficiency is $100 \%$. The bacterial and viral filtration efficiency is mainly dependent upon the basic weight and thickness of Poly Tetra Fluoro Ethylene (PTFE) nano fiber membrane and increased with increasing the basic weight or thickness of PTFE membrane. The filtration efficiency decreases only $4-5 \%$ after washing 20 times under the procedure of soaking for 10 minutes in cold water with a reasonable amount of detergent, gently crumbling with the hand 10 times, rinsing and naturally drying in free air. It is clearly shown that the product is filtration effective, washable and health care while maintaining the filtration and warming performance. For safety purpose, this product complies the regulation of CE and UL, FCC, and is certified by SGS for hazardous material. By a stylish fashion design, thoughtful structure design and choice of fabric appearance, this product also provides advantage of stylishness, robust design, user friendliness and high reusability to meet various customer need and to keep the nose and respiratory system in a healthy environment. Customer response proves the acceptance in the domestic and international market. Furthermore, the heating element is provided from the movable power supply with $10500 \mathrm{~mA}$. Hr and 1.0A above, which could be used for over 10 hours as the surface temperature controlled at $40{ }^{\circ} \mathrm{C} \pm 0.5{ }^{\circ} \mathrm{C}$. The LED lighting element is fixed around the front cheek so as to have a lighting, and warning effect safely when the wearers walked around a dark environment. The end applications of the thermal electrical device could be also set onto scarf, under wear, short pants, socks, hat, bed clothes, pillow cover, sleeve, mask, vest, jacket and jeans etc. during winter seasons [6-8].

\section{Conclusion}

o In order to overcome the serious allergens, dust mite and epidemics healthy problems, the electrical thermal mask with high performance filtration and health care functions has been proposed successfully.

o The filter media of this novel mask has been certificated by Nelson Lab. of the USA, both of the bacteria filtration efficiency and viral filtration efficiency are $99.9 \%$ and also tested with different particle size of between 7.0-0.4 micron by Taiwan Textile Research Institute (TTRI) in Taiwan, the filtration efficiency is $100 \%$.

o The functions of mask have air permeability, comfort, light and soft, fare infrared radiation, ultra-performance filtration, and moisturizing etc. and it can reuse, easy storage, durability, health care, washable, safety, and electrical warning advantages.

o The bacterial and viral filtration efficiency is dependent upon the basic weight or thickness of Poly Tetra Fluoro Ethylene (PTFE) nano fiber membrane and increased with increasing the basic weight or thickness of PTFE membrane.

o The temperature of electrical thermal mask can be controlled by MCU which could be used for over 10 hours as the surface temperature controlled at $40{ }^{\circ} \mathrm{C} \pm 0.5{ }^{\circ} \mathrm{C}$.

\section{References}

1. Hu YJ, Liao WS, Lin JC, Cheng KB - Hot compression mask, Taiwan Invention Patent Pending.

2. Hu YJ - High Performance Mask, Taiwan Patent M505944, Mainland China Patent 4629550.

3. Liao WS, Lin JC, Cheng KB (2017) Hot Compression Bra. Taiwan Invention Patent, No. I590773.

4. Kuo BC, Chen JC, Huang FL, Chang JT, Liu JY (2015) Effects of Fabric Structures and Yarn Constitutions on the Functional Properties of Cooling Knitted Fabric, $13^{\text {th }}$ Asian Textiles Conference, Geelong, Victoria, Australia, ID\# 157: 11.

5. Kuo YC, Cheng KB (2011) Cooling Bra: Fabrication Methods and Thermal Image Analysis. International Textile and Apparel Association, Philadelphia, Sheraton Hotel, USA Special Topic 12: 1-5. 
6. Cheng KB, Alvin YJ Hu, Liao WS (2016) Washable 3 Dimensional Mask with High Performance Filtration and Electrical Thermal Functions. Taipei International Invention Show and Techno mart.

7. Cheng KB, Alvin YJH, Liao WS (2016) Washable 3 Dimensional Mask with High Performance Filtration and Electrical Thermal Functions, Kaoshung International Invention and Design EXPO (KIDE).
8. Cheng KB, Alvin YJH, Liao WS (2017) Washable 3 Dimensional Mask with Optical, High Performance Filtration and Electrical Thermal Functions, Taipei International Invention Show and Techno mart.

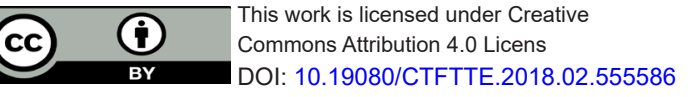

